\title{
The Association between Databases for Indexing Studies Intended for an Exercise Meta-Analysis of Arthritis Randomized Controlled Trials
}

\author{
George A. Kelley and Kristi S. Kelley \\ Meta-Analytic Research Group, School of Medicine, Department of Community Medicine, Robert C. Byrd Health Sciences Center, \\ West Virginia University, P.O. Box 9190, Morgantown, WV 26506-9190, USA \\ Correspondence should be addressed to George A. Kelley, gkelley@hsc.wvu.edu
}

Received 26 May 2012; Accepted 4 July 2012

Academic Editor: Ruben Burgos-Vargas

Copyright ( 2012 G. A. Kelley and K. S. Kelley. This is an open access article distributed under the Creative Commons Attribution License, which permits unrestricted use, distribution, and reproduction in any medium, provided the original work is properly cited.

Objective. The purpose of this study was to determine the database indexing of randomized controlled trials (RCTs) for a metaanalysis addressing the effects of exercise on pain and physical function in adults with arthritis and other rheumatic diseases (AORD). Methods. The number, percentage, and 95\% confidence intervals (CIs) for included articles at initial and follow-up periods were calculated from PubMed, EMBASE, CENTRAL, CINAHL, SPORTDiscus, and DAO databases. The number needed to review (NNR) was also calculated along with the number of articles retrieved by expert review. Cross-referencing from reviews and included articles also occurred. Results. Thirty-four of 36 articles (94.4\%, 95\% CI, 81.3-99.3) were located by database searching. PubMed and CENTRAL yielded 32 of 36 articles (88.9\%, 73.9-96.9). Two articles not identified in any of the other databases were found in either CINAHL or SPORTDicsus. Two other articles were located by scanning the reference lists of review articles. The NNR ranged from 2 (CINAHL) to 118 (SPORTDiscus). More articles were identified in EMBASE at follow-up (36\%, 12.142.2 versus $86.1 \%$, 70.5-95.3). Conclusions. Searching multiple databases and cross-referencing from reviews was important for identifying RCTs addressing the effects of exercise on pain and physical function in adults with AORD.

\section{Introduction}

Systematic reviews, with or without meta-analysis, are increasingly used to examine the effects of exercise in participants with arthritis and other rheumatic diseases (AORD). For example, a recent PubMed search up to May 25, 2012 and limited to systematic reviews and meta-analyses addressing the effects of exercise in participants with AORD yielded a total of 193 citations. One of the major steps when conducting a systematic review, with or without a meta-analysis, is a comprehensive search for all relevant articles on the topic of interest [1]. When conducting such a search, it is important to be thorough but efficient. Since the vast majority of articles included in systematic reviews are retrieved from electronic database searches [2,3], knowledge regarding the indexing of articles from each database is important. Recent research that examined previous meta-analyses of randomized controlled trials (RCTs) of the orthopedic surgical literature reported that more than $97 \%$ of included articles were retrieved from three electronic databases: MEDLINE, EMBASE, and Cochrane [2]. However, such results are likely to vary depending on the topic of choice. To the best of our knowledge, no previous study has attempted to assess the indexing of included articles for an exercise and AORD meta-analysis. This is surprising given the large number of meta-analytic citations on this topic. The authors have recently compiled a database of articles for a meta-analysis of RCTs addressing the effects of exercise on pain and physical function in adults with arthritis and other rheumatic diseases (AORDs). The purpose of this study was to determine the percentage of included articles that were indexed in the various databases. 


\section{Materials and Methods}

Articles included in the meta-analysis had to be (1) RCTs with an exercise-only group (aerobic, strength training, or both), (2) community-deliverable exercise interventions $\geq 4$ weeks, (3) a comparative control group (non-intervention, usual care, attention control), (4) adults aged 18 years and older with either rheumatoid arthritis, osteoarthritis, fibromyalgia, lupus, gout, or ankylosing spondylitis, (5) studies published in peer-reviewed journals as well as master's theses and dissertations, (6) articles published in any language between January 1, 1980 and January 1, 2008, and (7) data available for pain and/or physical function.

Articles were retrieved by (1) searching six different electronic databases (PubMed, EMBASE, Cochrane Central Register of Controlled Clinical Trials (CENTRAL), Cumulative Index to Nursing and Allied Health Literature (CINAHL), SPORTDiscus and Dissertation Abstracts Online (DAO), (2) cross-referencing from included articles, (3) cross-referencing from review articles, and (4) expert review (Dr. Miriam Nelson, Tufts University, personal communication, June 13, 2008). We included DAO because we were interested in studies that may not have been published but were available in thesis or dissertation format. We excluded Google Scholar based on a search that resulted in 25,500 citations after excluding patents and including only biology and medicine in the search. The rationale for this exclusion was based on fact that screening such a large number of citations was beyond the resources available for this and most other projects.

While the keywords and combination of keywords varied depending upon the database being searched, terms commonly used included "exercise," "arthritis," "rheumatic," "rheumatoid arthritis," "osteoarthritis," "fibromyalgia," "lupus," "gout," and "ankylosing spondylitis." A list of the user queries for each database is shown in (Supplement 1 available online at doi:10.1155/2012/624830.) With the exception of EMBASE, initial searches were conducted by the second author. For EMBASE, the initial search was conducted by an expert on exercise and arthritis who had access to such (Dr. Jennifer Hootman, personal communication, January 22, 2008).

Studies were selected for inclusion by both authors, independent of each other. The authors then met and reviewed every selection. Discrepancies were resolved by consensus. If consensus could not be reached, two experts on exercise and AORD acted as arbitrators (Dr. Jennifer Hootman and Dr. Dina Jones, personal communication). Using Cohen's kappa statistic, the overall agreement rate prior to correcting discrepancies was 0.89 .

For this small study, the number, percentage, and 95\% confidence intervals for included articles derived from the initial search of each database was calculated. As a followup, the number, percentage, and 95\% confidence intervals for articles actually indexed in each database was also calculated. This was accomplished by entering various combinations of each missing article's title, author name(s), journal name, and publication year into each database [4]. For the DAO database, only the one included dissertation was searched for [5]. With the exception of EMBASE, the identification of articles at follow-up was conducted by the first author. Follow-up searching in EMBASE was conducted by an expert on exercise and arthritis (Dr. Jennifer Hootman, personal communication, December 30, 2009).

In addition to calculating the number, percentage, and 95\% confidence intervals for included articles from initial and follow-up searches, the number needed to review (NNR) was calculated for each database by dividing the total number of initial citations to be screened by the number of articles that were included.

\section{Results}

A flow diagram describing the initial search process, including the number of studies examined, is shown in Figure 1. The results for initial and follow-up searches for included studies as well as the NNR are shown in Table 1, the reference list of included articles in Supplement 2 available online at doi:10.1155/2012/624830, and database indexing for each article from each database in Supplement 3 available online at doi:10.1155/2012/624830.

3.1. Initial Search. Thirty-four of 36 articles $(94.4 \%, 95 \%$ CI, 81.3-99.3) that met the criteria for inclusion were located via initial database searches. Two articles $(5.6 \%, 0.7-18.7)$ were located by scanning the reference lists of review articles $[6,7]$. None were included based on scanning the reference lists of included articles or from expert review. All included articles were published in the English language. As can be seen in rows 1 through 3 of Table 1, the CENTRAL and PubMed databases yielded the greatest number of included articles followed by EMBASE, CINAHL, and SPORTDiscus. None were located using DAO. All studies initially identified in EMBASE were also identified in PubMed and/or CENTRAL. When combined, both the CENTRAL and PubMed databases yielded 32 of 36 articles $(88.9 \%, 73.9-96.9)$. The crossover between the two databases was $75.0 \%$ (56.6-88.5). Excluding the two articles that were located by scanning the reference lists of reviews $[6,7]$, one article that was not available in PubMed, CENTRAL, or EMBASE was located using CINAHL [8] while another was identified using SPORTDiscus [9].

3.2. Follow-Up Searches. When each database was searched for each article at follow-up (rows 4 through 6 of Table 1), the CENTRAL and PubMed databases yielded the greatest number of articles followed by EMBASE, CINAHL, and SPORTDiscus. With the exception of DAO, all follow-up searches resulted in the identification of a greater number of articles. This was particularly true for EMBASE (nonoverlapping confidence intervals and $50.1 \%$ difference). For the two articles that were initially identified by scanning the reference lists of review articles but not found in any database $[6,7]$, one [6] was found in PubMed, EMBASE, CENTRAL, and CINAHL while the other [7] was located in PubMed, EMBASE, and CENTRAL. One article that was initially identified in SPORTDiscus was also found in 

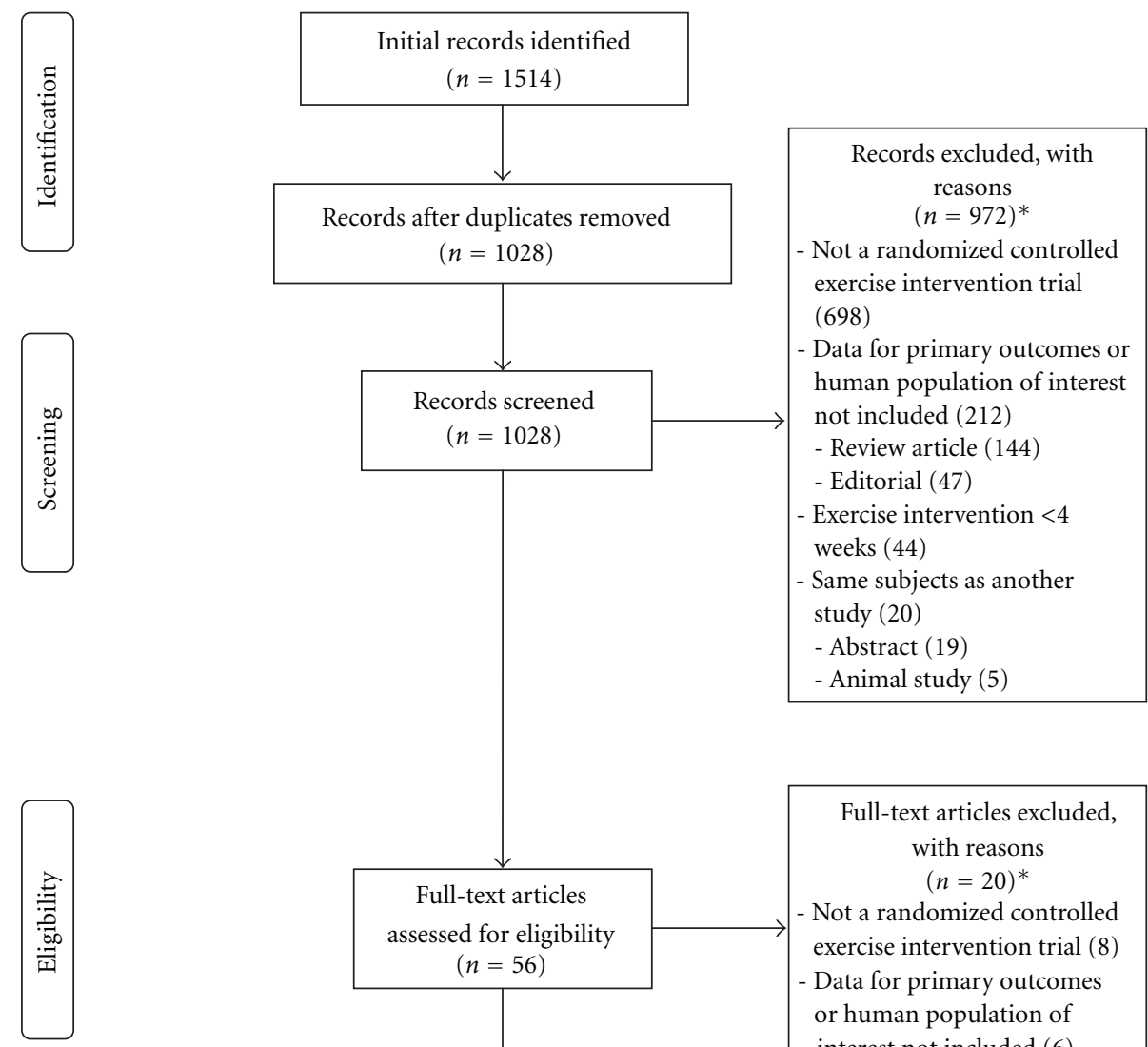

Full-text articles excluded, with reasons $(n=20)^{*}$

- Not a randomized controlled exercise intervention trial (8) - Data for primary outcomes or human population of interest not included (6)

- Same subjects as another study (6)

- Review article (3)

Articles included $(n=36)$

- Editorial (1)

FIGURE 1: Flow diagram describing the search process. ${ }^{*}$, number of reasons exceeds the number of studies excluded because some studies excluded for more than one reason.

TABLE 1: Indexing of articles from database searches.

\begin{tabular}{|c|c|c|c|c|c|c|}
\hline Variable & PubMed & EMBASE & CENTRAL & CINAHL & SPORTDiscus & $\mathrm{DAO}$ \\
\hline \multicolumn{7}{|l|}{ Initial search } \\
\hline$n(\%)$ & $27(75)$ & $9(36)$ & $29(80.6)$ & $11(30.6)$ & $3(8.3)$ & $0(0)$ \\
\hline$(95 \% \mathrm{CI})$ & $(57.8-87.9)$ & $(12.1-42.2)$ & $(64.0-91.8)$ & $(16.3-48.1)$ & $(1.8-22.5)$ & (NA) \\
\hline \multicolumn{7}{|c|}{ Follow-up search } \\
\hline$n(\%)$ & $34(94.4)$ & $31(86.1)$ & $35(97.2)$ & $19(52.8)$ & $6(16.7)$ & $0(0)$ \\
\hline$(95 \% \mathrm{CI})$ & $(81.3-99.3)$ & $(70.5-95.3)$ & (85.5-99.9) & $(35.5-69.6)$ & $(6.4-32.8)$ & (NA) \\
\hline NNR & 9 & 12 & 16 & 2 & 118 & NA \\
\hline
\end{tabular}

Notes: Total number of articles included = 36; CENTRAL: Cochrane Central Register of Controlled Clinical Trials; CINAHL: Cumulative Index to Nursing and Allied Health Literature; DAO: Dissertation Abstracts Online; $n$ (\%): number and percentage of articles; $95 \%$ CI: $95 \%$ confidence interval; NNR: Number needed to review, calculated by dividing the total number of citations to review per database divided by the number of studies included from the initial search; NA: not applicable.

PubMed, EMBASE, CENTRAL, and CINAHL at follow-up [9]. One other article continued to be limited in location to CINAHL [8].

3.3. Number Needed to Review (NNR). Row 7 of Table 1 includes data on the NNR. As shown, the largest NNR was from the SPORTDiscus database while the smallest was from CINAHL.

\section{Discussion}

The results of this study suggest that searching multiple databases was important when trying to locate all RCTs 
addressing the effects of exercise on AORD in adult humans. This suggestion is based on the lack of $100 \%$ crossover between databases as well as the inability to locate, initially, articles that may reside in multiple databases but may only be identified by one. In addition, because our search strategies were unable to identify articles that were located in one or more databases but could not be identified by any of them suggests that scanning the reference lists of resources such as review articles was important. The inability to initially locate articles that existed in a database, especially EMBASE, was most likely the result of the queries developed and used to conduct the searches and/or the search filters provided by the database vendors. In the case of EMBASE, that large discrepancy in this study may have been the result of someone with less experience conducting the searches.

In contrast to database searching and scanning the reference lists of review articles, we did not find expert review or scanning the reference lists of included articles to be helpful. Given these findings, inclusion of these approaches may not be worthwhile. However, before any firm conclusions can be drawn, this should probably be tested using other systematic reviews.

Clearly, the two most important databases searched for the current study were CENTRAL and PubMed. While including EMBASE has been recommended [2], it did not yield any studies that could not be identified in CENTRAL and/or PubMed. However, since the focus of CENTRAL is on RCTs, it is unlikely to be very helpful for non-RCTs such as studies of test accuracy [3].

With the exception of SPORTDiscus, the NNR appeared to be equitable for all other databases. Based on these findings, it might seem appropriate to suggest that SPORTDiscus not be searched given the large NNR and low yield. However, since the goal of a systematic review is to identify all articles that meet one's inclusion criteria and one article not initially identifiable by any other databases or approaches was located using SPORTDiscus [9], the inclusion of this database was important. In contrast, the inclusion of the DAO database was not important for this study.

Given that systematic reviews are increasingly used in the exercise and arthritis literature, thorough and efficient literature searches are important for conducting high-quality systematic reviews with or without meta-analysis. The results of the current study provide an important first step in that direction.

While the current study provides important information in relation to searching and retrieving exercise studies with respect to $\mathrm{AORD}$, the generalization of the current findings to other systematic reviews on AORD may be limited. Additional research on optimal approaches in searching for relevant articles to include in systematic reviews on AORD is needed. For a much broader perspective on this important topic, one is referred to the recent work of others in relation to optimal search strategies for the identification and retrieval of studies for systematic reviews, with or without meta-analysis [10-17].

\section{Conclusions}

The results of this study suggest that searching multiple databases as well as cross-referencing from review articles was important for identifying RCTs addressing the effects of exercise on pain and physical function in adults with AORD. However, additional research in this area is needed before any firm recommendations can be made.

\section{Disclosure}

The authors have nothing to disclose.

\section{Disclaimer}

The findings and conclusions in this article are those of the authors and do not necessarily represent the views of the Centers for Disease Control and Prevention.

\section{Acknowledgments}

This work is supported under a cooperative agreement from the Centers for Disease Control and Prevention through the Association of American Medical Colleges, grant number U36/CCU319276, AAMC ID number MM-0944-06/06.

\section{References}

[1] J. P. T. Higgins and S. Green, Cochrane Handbook for Systematic Reviews of Interventions (version 5.0.2), Wiley-Blackwell, 2009.

[2] G. P. Slobogean, A. Verma, D. Giustini, B. L. Slobogean, and K. Mulpuri, "MEDLINE, EMBASE, and Cochrane index most primary studies but not abstracts included in orthopedic meta-analyses," Journal of Clinical Epidemiology, vol. 62, no. 12, pp. 1261-1267, 2009.

[3] P. Whiting, M. Westwood, M. Burke, J. Sterne, and J. Glanville, "Systematic reviews of test accuracy should search a range of databases to identify primary studies," Journal of Clinical Epidemiology, vol. 61, no. 4, pp. 357-364, 2008.

[4] M. L. van Driel, A. De Sutter, J. De Maeseneer, and T. Christiaens, "Searching for unpublished trials in Cochrane reviews may not be worth the effort," Journal of Clinical Epidemiology, vol. 62, no. 8, pp. 838-844, 2009.

[5] W. Eungpinichpong, The efficacy of physical exercise programmes for patients with osteoarthritis of the knee as determined by clinical and gait parameters [dissertation], University of Otago, Dunedin, New Zealand, 1998.

[6] M. Fransen, L. Nairn, J. Winstanley, P. Lam, and J. Edmonds, "Physical activity for osteoarthritis management: a randomized controlled clinical trial evaluating hydrotherapy or Tai Chi classes," Arthritis Care and Research, vol. 57, no. 3, pp. 407414, 2007.

[7] D. Munguía-Izquierdo and A. Legaz-Arrese, "Exercise in warm water decreases pain and improves cognitive function in middle-aged women with fibromyalgia," Clinical and Experimental Rheumatology, vol. 25, no. 6, pp. 823-830, 2007.

[8] J. Wessel and H. A. Quinney, "Pain experienced by persons with rheumatoid arthritis during isometric and isokinetic exercise," Physiotherapy Canada, vol. 36, no. 3, pp. 131-134, 1984. 
[9] D. S. Nichols and T. M. Glenn, "Effects of aerobic exercise on pain perception, affect, and level of disability in individuals with fibromyalgia," Physical Therapy, vol. 74, no. 4, pp. 327332, 1994.

[10] M. Kastner, N. L. Wilczynski, A. K. McKibbon, A. X. Garg, and R. B. Haynes, "Diagnostic test systematic reviews: bibliographic search filters ("Clinical Queries") for diagnostic accuracy studies perform well," Journal of Clinical Epidemiology, vol. 62, no. 9, pp. 974-981, 2009.

[11] K. A. McKibbon, N. L. Wilczynski, and R. B. Haynes, "Retrieving randomized controlled trials from medline: a comparison of 38 published search filters," Health Information and Libraries Journal, vol. 26, no. 3, pp. 187-202, 2009.

[12] N. L. Wilczynski and R. B. Haynes, "Consistency and accuracy of indexing systematic review articles and meta-analyses in medline," Health Information and Libraries Journal, vol. 26, no. 3, pp. 203-210, 2009.

[13] N. L. Wilczynski, A. X. Garg, and B. Haynes, "A method for defining a journal subset for a clinical discipline using the bibliographies of systematic reviews," Studies in Health Technology and Informatics, vol. 12, no. 1, pp. 721-724, 2007.

[14] N. L. Wilczynski and R. B. Haynes, "EMBASE search strategies achieved high sensitivity and specificity for retrieving methodologically sound systematic reviews," Journal of Clinical Epidemiology, vol. 60, no. 1, pp. 29-33, 2007.

[15] S. S. L. Wong, N. L. Wilczynski, and R. B. Haynes, "Comparison of top-performing search strategies for detecting clinically sound treatment studies and systematic reviews in MEDLINE and EMBASE," Journal of the Medical Library Association, vol. 94, no. 4, pp. 451-455, 2006.

[16] S. S. L. Wong, N. L. Wilczynski, and R. B. Haynes, "Optimal CINAHL search strategies for identifying therapy studies and review articles: health policy and systems," Journal of Nursing Scholarship, vol. 38, no. 2, pp. 194-199, 2006.

[17] N. L. Wilczynski, D. Morgan, and R. B. Haynes, "An overview of the design and methods for retrieving high-quality studies for clinical care," BMC Medical Informatics and Decision Making, vol. 5, article 20, 2005. 


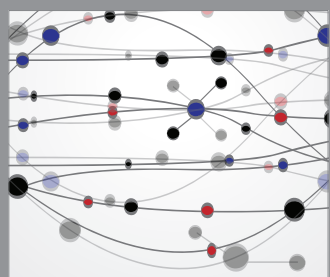

The Scientific World Journal
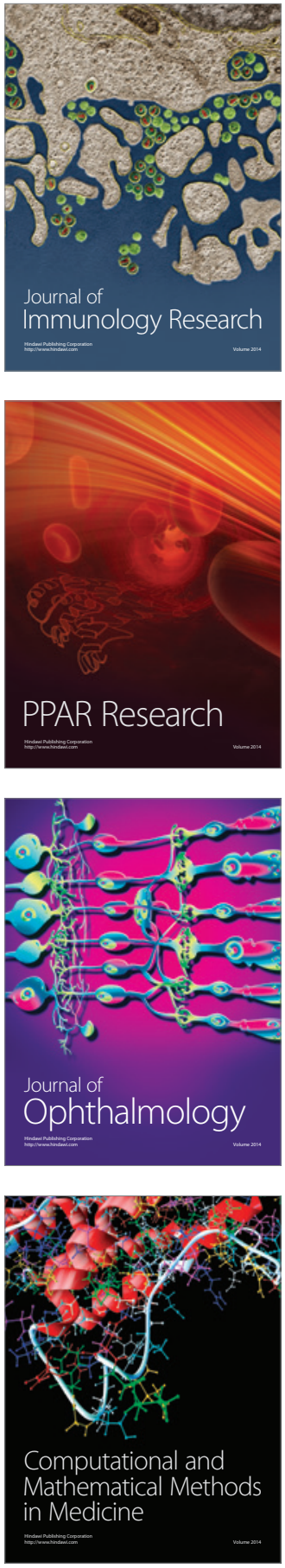

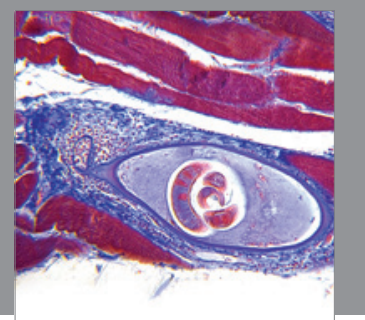

Gastroenterology

Research and Practice
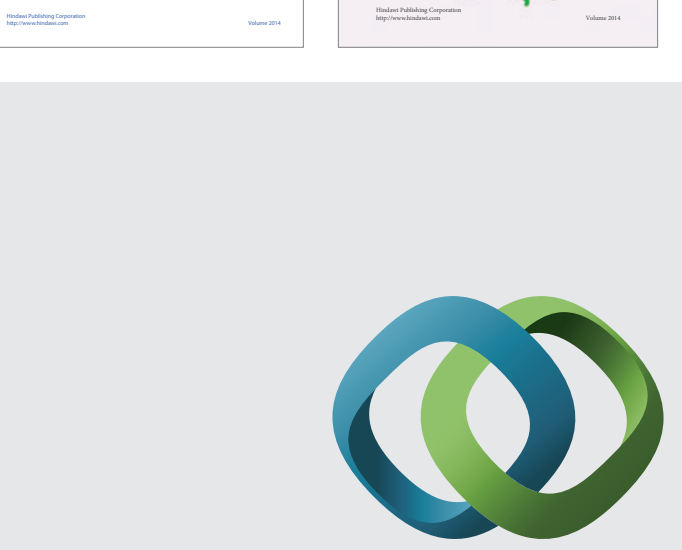

\section{Hindawi}

Submit your manuscripts at

http://www.hindawi.com
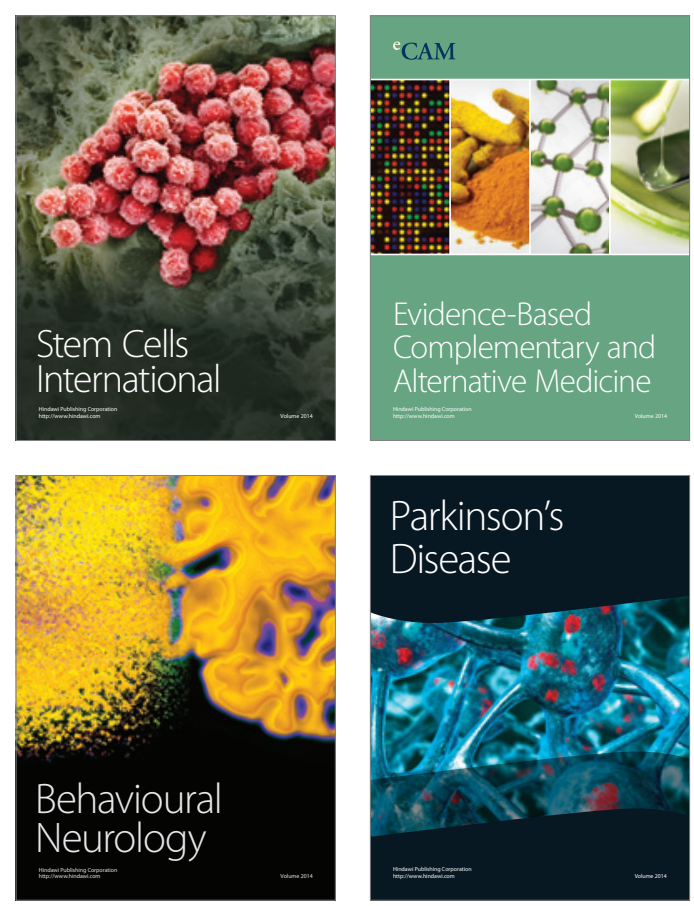

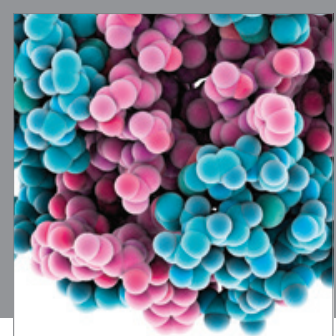

Journal of
Diabetes Research

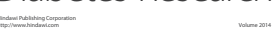

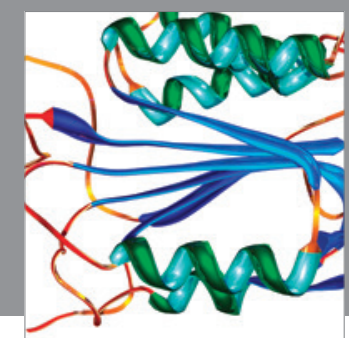

Disease Markers
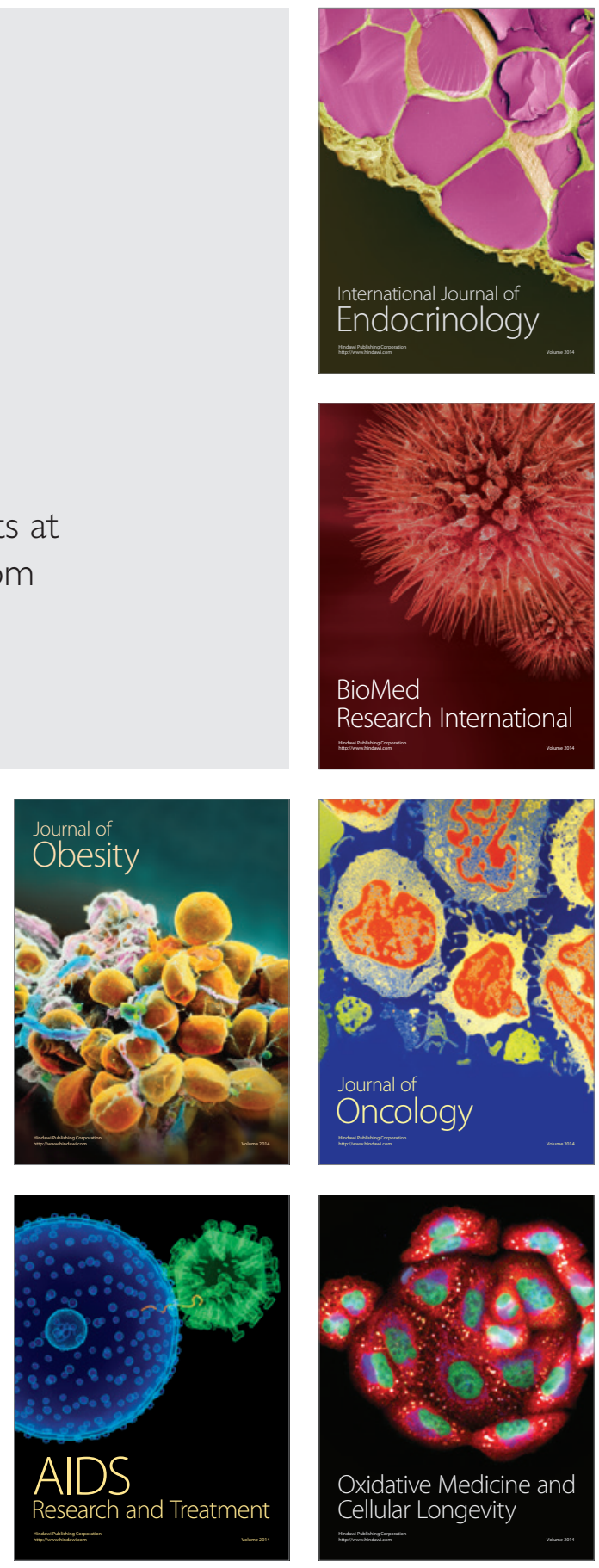